hep-ph/9502387

YCTP-P1-95A

\title{
A strongly first order electroweak phase transition from strong symmetry-breaking interactions
}

\author{
Thomas Appelquist, Myckola Schwetz ${ }^{\dagger}$ and Stephen B. Selipsky ${ }^{\ddagger}$ \\ Department of Physics, Yale University, New Haven, Connecticut 06520-8120
}

February 23, 1995

\begin{abstract}
We argue that a strongly first order electroweak phase transition is natural in the presence of strong symmetry-breaking interactions, such as technicolor. We demonstrate this using an effective linear scalar theory of the symmetry-breaking sector.
\end{abstract}

PACS numbers: 11.30.Qc, 11.10.Wx, 11.30.Rd, 12.60.Nz

\section{Introduction}

The nature of the finite temperature electroweak phase transition remains an important and elusive problem in particle physics. The possibility of electroweak scale baryogenesis, which has helped generate new interest in this subject [1], requires the transition to be strongly first order; only then does the universe depart from thermal equilibrium, satisfying the third of Sakharov's requirements for baryogenesis and avoiding the washing out of any generated baryon asymmetry. This paper investigates the electroweak phase transition when the symmetry-breaking sector involves strong interactions, such as technicolor. We use an effective scalar theory to describe these interactions, and find that the transition is first order if this sector exhibits a $U(N)_{L} \times U(N)_{R}$ global symmetry. When the interactions are relatively strong, the transition is strongly first order: the discontinuity in the order parameter at the critical temperature is of the order of the zero temperature expectation value $v$.

\footnotetext{
*Electronic address: twa@genesis3.physics.yale.edu

${ }^{\dagger}$ Electronic address: ms@genesis2.physics.yale.edu

${ }^{\ddagger}$ Electronic address: stephen@genesis1.physics.yale.edu
} 
To set the stage, we briefly review what is known about the electroweak phase transition in the minimal standard model. A variety of studies of the finite temperature effective potential have addressed this problem, using methods including the $\epsilon$ expansion, Wilson-effective-action and "average action" renormalization group techniques, or graphical summation schemes with names such as daisy and superdaisy [2, 3]. They allow improved treatments of the infrared problems [4] that can arise near phase transitions. The transition appears to be first order when the Higgs boson is lighter than the $W$ and $Z$, but at most rather weakly so for an experimentally acceptable Higgs boson mass. As that mass increases, corresponding to stronger scalar coupling, the strength of the transition decreases [5]. When the Higgs boson is much heavier than the $W$ and $Z$, it is reasonable to neglect the gauge and Yukawa interactions when studying the transition. In that approximation the minimal standard model is the $O(4)$ linear sigma model, and the chiral transition in $O(N)$ models seems to be second order or at most weakly first order [6, 7, 8].

Beyond the minimal standard model, less is known about the nature of the electroweak phase transition, especially if the symmetry-breaking sector is strongly interacting, as in a technicolor theory. Lattice studies [9] of QCD-like gauge theories indicate that with two light fermions, corresponding to an $S U(2)_{L} \times S U(2)_{R} \sim O(4)$ global symmetry, the transition is second order, whereas with more light fermions and a larger global symmetry, the transition is first order. This suggests that technicolor theories, which often do have more than two technifermions, might lead to a first order transition. To complement the lattice results, we analyze the strongly coupled symmetrybreaking problem using an effective scalar theory, which is partly amenable to analytic treatment and avoids some of the uncertainties associated with lattice techniques.

\section{The Linear Sigma Model}

The properties of the phase transition depend on the dynamics of the electroweak order parameter. We assign it to transform under a global $U(N)_{L} \times U(N)_{R}$ chiral symmetry, motivated by technicolor theories, in which the order parameter is a condensate of fermion bilinears. A one family technicolor model for example corresponds to $N=8$ flavors, large enough to suggest utility of the large $N$ 
expansion in the analysis. The most economical effective scalar theory of electroweak interactions is of course the nonlinear chiral Lagrangian. To allow for the existence of a phase transition above which the order parameter vanishes, however, we must go beyond the nonlinear theory [10]. Accordingly, we will use an effective linear sigma model to describe the symmetry-breaking sector [11, 12]. Neglecting standard model gauge and fermion interactions in comparison to this sector's strong interactions, we take the Lagrangian to be

$$
\mathcal{L}=\operatorname{Tr}\left(\partial_{\mu} \Sigma^{\dagger} \partial^{\mu} \Sigma\right)-\mu^{2} \operatorname{Tr}\left(\Sigma^{\dagger} \Sigma\right)-\frac{\lambda_{1}}{4 N^{2}}\left(\operatorname{Tr} \Sigma^{\dagger} \Sigma\right)^{2}-\frac{\lambda_{2}}{4 N} \operatorname{Tr} \mathcal{O}_{2}^{2}
$$

Here we have defined the traceless operator $\mathcal{O}_{2} \equiv \Sigma^{\dagger} \Sigma-(\mathcal{I} / N) \operatorname{Tr}\left(\Sigma^{\dagger} \Sigma\right)$, where

$$
\Sigma=\left(\sigma_{a}+i \pi_{a}\right) T^{a} \quad\left(a=0,1, \ldots, N^{2}-1\right)
$$

with generator matrix normalization $2 \operatorname{Tr}\left(T^{a} T^{b}\right)=\delta^{a b}$ and $T^{0}=\mathcal{I} / \sqrt{2 N}$. Higher-dimensional (nonrenormalizable) interactions could also be included in the effective theory, but we argue in section 4 that their omission does not qualitatively affect our conclusions.

In order that the tree level potential be bounded below we must have $\lambda_{1}>0$, and either $\lambda_{2}>0$, or $\lambda_{2}<0$ with $\lambda_{1}>(N-1)\left|\lambda_{2}\right|$. Spontaneous symmetry-breaking then occurs for negative $\mu^{2}$. We do not consider the $\lambda_{2}<0$ case, in which $U(N)_{L} \times U(N)_{R}$ breaks to $[U(N-1) \times U(1)]_{L} \times[U(N-$ 1) $\times U(1)]_{R}$. For $\lambda_{2}>0$ the breaking pattern is $U(N)_{L} \times U(N)_{R} \rightarrow U(N)_{V}$, and the tree level potential at zero temperature is minimized by a vacuum expectation value $\langle\Omega|\Sigma| \Omega\rangle$ that can be taken, after a $U(N)_{L} \times U(N)_{R}$ transformation, to be real and proportional to the identity matrix:

$$
\begin{aligned}
\left\langle\Omega\left|\sigma_{0}\right| \Omega\right\rangle^{2} & =\frac{4 N^{2}}{\lambda_{1}}\left|\mu^{2}\right|, \\
\left\langle\Omega\left|\sigma_{a}\right| \Omega\right\rangle & =0 \quad\left(a=1, \ldots, N^{2}-1\right), \\
\left\langle\Omega\left|\pi_{a}\right| \Omega\right\rangle & =0 \quad\left(a=0, \ldots, N^{2}-1\right) .
\end{aligned}
$$

The $\lambda_{2}$ independence of $\left\langle\Omega\left|\sigma_{0}\right| \Omega\right\rangle$ follows from the definition of $\mathcal{O}_{2}$. The $W$ mass is given by $\frac{1}{2} g\left\langle\sigma_{0}\right\rangle$, so that $\left\langle\Omega\left|\sigma_{0}\right| \Omega\right\rangle=v \equiv 250 \mathrm{GeV}$. The $2 N^{2}$ degrees of freedom described by the $\Sigma$ fields correspond to $N^{2} \mathrm{Nambu}-$ Goldstone scalars $\pi_{a}$, and $N^{2}$ scalars $\left(\sigma_{a}, \sigma_{0}\right)$ that describe the massive fluctuations 
of the order parameter. Their masses

$$
m_{\pi_{a}}^{2}=0, \quad m_{\sigma_{a}}^{2}=\frac{\lambda_{2}}{2 N^{2}} v^{2}, m_{\sigma_{0}}^{2}=\frac{\lambda_{1}+\lambda_{2}}{2 N^{2}} v^{2}
$$

scale as $1 / N$ relative to $v=250 \mathrm{GeV}$.

Among the $N^{2}$ Nambu-Goldstone scalars, three become the longitudinal $W^{ \pm}$and $Z$, the $\pi_{0}$ gains mass via anomalous breaking of the axial $U(1)$, and the remaining $\left(N^{2}-4\right)$ are pseudoNambu-Goldstone bosons, all of which gain small masses from neglected standard model and other (Extended Technicolor) interactions. We neglect these pseudo-Nambu-Goldstone masses. The anomaly-generated $\pi_{0}$ mass could also be implemented in our effective Lagrangian, by adding a nonrenormalizable (for $N>4$ ) determinantal interaction [11, 12, 13], but omission of this term affects the masslessness of only one mode and will not change our conclusions. In the limit $\lambda_{2}=0$, the symmetry of the theory increases from $U(N) \times U(N)$ to $O\left(2 N^{2}\right)$ and the spontaneous breaking produces $\left(2 N^{2}-1\right)$ Nambu-Goldstone bosons, with only the $\sigma_{0}$ massive.

The factors of $N$ in Eq. (1) allow a nontrivial large $N$ expansion holding $\lambda_{1}$ and $\lambda_{2}$ fixed. In the $\lambda_{2}=0$ limit, the leading $1 / N$ approximation is tractable, corresponding to the familiar linear bubble sum. With $\lambda_{2}$ nonzero, on the other hand, all planar diagrams involving the $\lambda_{2}$ interaction contribute at leading order. For both interactions, the strong coupling limit sets in at $\lambda_{i} / 16 \pi^{2} \sim 1$, when higher loops are as large as lower order contributions. In this limit the scalar masses of Eq. (4) saturate the bound $4 \pi v / N$ [14].

Symmetry-breaking aspects of the $U(N) \times U(N)$ linear sigma model at zero temperature in various spatial dimensions have previously been examined using the $\epsilon$ expansion [1] combined with the effective potential [15], and with lattice methods [16]. These investigations found ColemanWeinberg [17] behavior: broken symmetry when $\mu^{2}$ is tuned to zero. The $\epsilon$ expansion analysis, using renormalization group flow in $d=4-\epsilon$ spatial dimensions, shows that a second order transition is not self-consistent for $N>2$ [11]. However, studying the transition as a function of Lagrangian parameters at $T=0$ is not the same as studying it as a function of temperature. Since effective three-dimensional theories apply to finite temperature only by assuming decoupling of nonstatic 
modes (discussed below), they are limited to the second order or very weakly first order cases. The same is true of analyses of the transition strength using renormalization group flow "time" [18]. Furthermore, the $\epsilon$ expansion may break down before $\epsilon=1$ or miss IR fixed points. Nevertheless, the above work does hint that a full finite temperature calculation might find a first order phase transition.

\section{Computing the Effective Potential}

We analyze the phase transition with the finite temperature effective potential defined in Euclidean periodic time, which describes the system in thermal equilibrium 19. Matsubara frequencies $k^{0}=(2 \pi n T)$, where $n=0, \pm 1, \pm 2, \ldots$, then appear in propagators. If the relevant momentum scales were much smaller than $2 \pi T$ (e.g. the high temperature limit), all but the $n=0$ modes would decouple, leaving an effective three-dimensional theory. This is not true at a strongly first order phase transition, where we shall see that relevant scales are of order $2 \pi T$. (The transition temperature will be of order $v / N$, while in the strongly coupled case the masses in Eq. (4) approach $4 \pi v / N$.) We will therefore retain the full four-dimensional dynamics; we will also avoid the relative simplicity of the high temperature expansion, relying below on numerical integration for explicit results.

We can obtain the finite temperature effective potential as the sum of one-particle-irreducible vacuum graphs in a background field [20]. For the linear sigma model of interest here, the calculation is conveniently implemented using the auxiliary field method [21, 22] to eliminate the $\lambda_{1}$ interaction in favor of a nonpropagating dimension-two field $\chi$. The auxiliary field facilitates resummation to all orders in $\lambda_{1}$, at each order in $1 / N$. It consists of adding to the Lagrangian a perfect square, which yields an irrelevant constant factor upon path integration over the auxiliary field:

$$
\mathcal{L} \rightarrow \mathcal{L}+\frac{4 N^{2}}{\lambda_{1}}\left(\chi-\mu^{2}-\frac{\lambda_{1}}{4 N^{2}} \operatorname{Tr}\left(\Sigma^{\dagger} \Sigma\right)\right)^{2}
$$

Before presenting results for the full theory, it is useful to review the nature of the phase tran-

sition in the $\lambda_{2}=0$ theory $\left(O\left(2 N^{2}\right)\right.$ symmetry). Analyses using the $1 / N$ expansion show that the 
high temperature phase transition here is second order [7, 22, 23]. The leading order computation of the effective potential corresponds to the "superdaisy" approximation [19, 24, 25], which can therefore be justified in the context of the $1 / N$ expansion. Without superdaisy resummation, on the other hand, a one-loop computation using the Lagrangian of Eq. (1) with $\lambda_{2}=0$ gives a first order result [3, 26]. This is unreliable, since the theory's infrared behavior is controlled by the effective loop expansion parameter, of order $\left(\lambda_{1} / 16 \pi^{2}\right) T / m(\sigma)$. Here $m^{2}(\sigma) \equiv \mu^{2}+\left(\lambda_{1} / 4\right) \sigma^{2}$ is the effective mass of the lightest excitations, in the quantum state $|\Psi\rangle$ with amplitude peaked at the classical background field $\left\langle\Psi\left|\sigma_{0}\right| \Psi\right\rangle \equiv N \sigma$. This mass vanishes for Nambu-Goldstone bosons at a symmetry-breaking minimum of the effective potential, so the perturbative expansion breaks down due to infrared divergences, in some $\sigma$ neighborhood of the minimum. The $1 / N$ expansion avoids this perturbative problem by a summation of graphs at each order in $1 / N$.

To leading order in $1 / N$, the $O\left(2 N^{2}\right)$ computation involves only a single loop of the $2 N^{2}-1$ Nambu-Goldstone bosons in the presence of background $\chi$ and $\sigma$ fields, using the Lagrangian of Eq. (5). Because the $\chi$ field is nondynamical, it can be eliminated in favor of $\sigma$ using the saddlepoint equation of motion. This procedure reveals the second order behavior. It is equivalent to using the Lagrangian of Eq. (何) and solving a momentum-independent Schwinger-Dyson equation for the Nambu-Goldstone boson effective mass, whose solution is inserted into the single loop of Nambu-Goldstone bosons.

The effective potential computed in this way becomes complex for $\sigma$ between the symmetrybreaking minimum (where the Nambu-Goldstone boson mass vanishes) and the origin. This occurs for $\lambda_{2} \neq 0$ as well; in either case, the imaginary part may be interpreted [27] as the transition amplitude for leaving an unstable configuration, into which a classical external source cannot force the system. One may define a real quantity by a Maxwell construction: the "convex effective potential" (the convex envelope of the real part of the above effective potential) [28]. Although the convex effective potential approximately describes the lowest energy quantum state for a given $\sigma$, if a local minimum remains at the origin then the system is likely to supercool, remaining in the quantum state peaked at $\sigma=0$ even below the temperature $T_{c}$ where the symmetry-breaking 
minimum falls to a lower energy than the symmetric one [29]. The phase transition is thus controlled by the appearance and location of minima in the nonconvex potential as computed here.

Turning now to the general case with $\lambda_{2} \neq 0$, the computation is complicated by the fact that planar graphs to all orders in $\lambda_{2}$ contribute at leading order in the $1 / N$ expansion. Auxiliary fields could also be introduced to replace the $\lambda_{2}$ interaction, but not very usefully since an infinite class of graphs would still contribute at each order in $1 / N$. We instead use the Lagrangian with only the auxiliary $\chi$ field, Eq. (5), and compute the effective potential as we did in the $\lambda_{2}=0$ case: one loop of $\left(2 N^{2}-1\right)$ quanta in background $\chi$ and $\sigma$ fields (Fig. 1), after which the auxiliary $\chi$ field is eliminated using its equation of motion. This approximation sums all orders in $\lambda_{1}$ to leading order in $1 / N$. It also includes the (tree-level) contribution of the $\lambda_{2}$ interaction to the effective masses of the $\left(2 N^{2}-1\right)$ quanta in the loop, lifting the degeneracy between the $N^{2}$ Nambu-Goldstone $\pi_{a}$ modes and the $\left(N^{2}-1\right) \sigma_{a}$ modes which are Nambu-Goldstone bosons only in the $\lambda_{2} \rightarrow 0$ limit. This approximation to the $U(N) \times U(N)$ theory should provide a reliable qualitative guide to the nature of the phase transition, even without contributions like those of Fig. 2 .

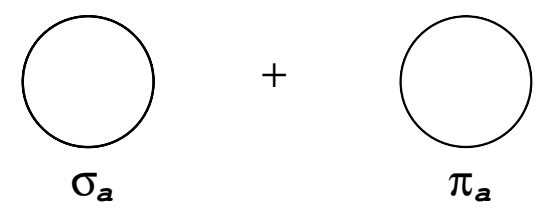

Figure 1: Feynman graphs included in our computation of $V_{\text {eff }}$. The vacuum loops of $\left(N^{2}\right)$ flavors of $\pi_{a}$ and $\left(N^{2}-1\right)$ of $\sigma_{a}$ propagate in background $\sigma_{0}$ and $\chi$ fields.

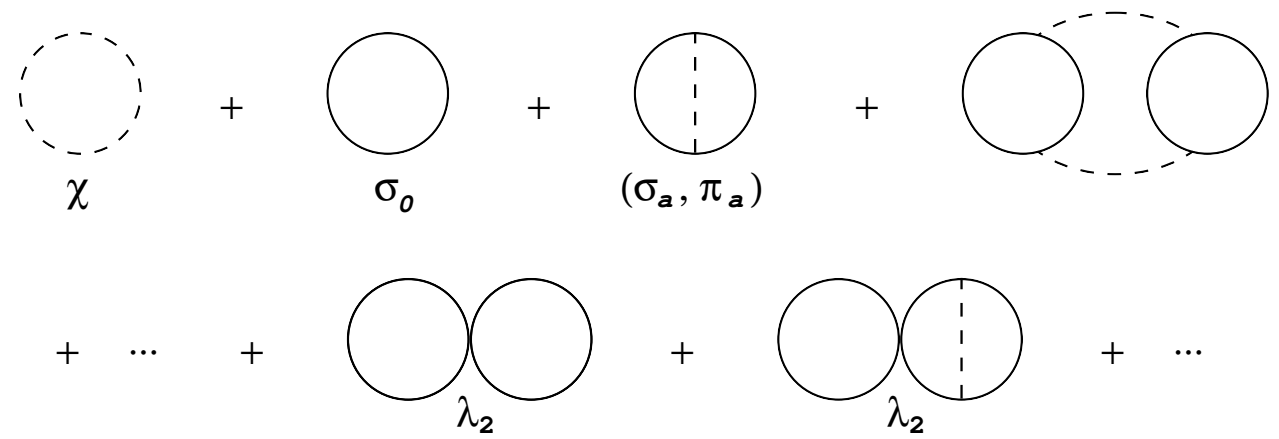

Figure 2: Some neglected graphs. One-loop graphs subleading in $1 / N$; and higher order graphs suppressed by powers of $1 / N$ or $\lambda_{2}$ or both. Dashed lines are diagonalized $\chi$ field propagators, solid lines are diagonalized $\sigma$ and $\pi$ fields. 
The computation requires renormalization counterterms which we define conventionally at zero temperature. Diagonalizing the propagators then gives the one-loop result for the finite temperature effective potential as a function of $\sigma \equiv\left\langle\Psi\left|\sigma_{0} / N\right| \Psi\right\rangle$ and $\chi \equiv\langle\Psi|\chi| \Psi\rangle$ :

$$
\begin{aligned}
N^{-2} V(\sigma, \chi) & =\frac{1}{2} \chi \sigma^{2}+2 \frac{\mu^{2} \chi}{\lambda_{1}}-\frac{\chi^{2}}{\lambda_{1}} \\
& +\frac{1}{2} \int_{T} \ln \left(k^{2}+\chi\right)+\frac{1}{2} \int_{T} \ln \left(k^{2}+\chi+\frac{\lambda_{2}}{2} \sigma^{2}\right) \\
& -(T=0 \text { counterterms })
\end{aligned}
$$

where $k^{0}=(2 \pi n T)$ and $\int_{T} \equiv T \int(2 \pi)^{-3} d^{3} k \sum_{n=-\infty}^{n=\infty}$. The first integral corresponds to the loop of $\pi_{a}$ (Nambu-Goldstone) scalars whose effective mass is $\chi$; the second integral corresponds to the loop of $\sigma_{a}$ scalars whose effective mass is $\chi+\left(\lambda_{2} / 2\right) \sigma^{2}$. The subtracted counterterms are

$$
\begin{aligned}
\int_{0} \ln k^{2} & +\int_{0} \frac{\chi+\frac{\lambda_{2}}{2 N^{2}} \operatorname{Tr}\left(\Sigma^{\dagger} \Sigma\right)}{k^{2}}-\frac{1}{4} \int_{0} \frac{\chi^{2}+\left[\chi+\frac{\lambda_{2}}{N^{2}} \operatorname{Tr}\left(\Sigma^{\dagger} \Sigma\right)\right]^{2}}{\left(k^{2}+M_{r}^{2}\right)^{2}} \\
& =\int_{0} \ln k^{2}+\int_{0} \frac{\chi+\frac{\lambda_{2}}{4} \sigma^{2}}{k^{2}}-\frac{1}{4} \int_{0} \frac{\chi^{2}+\left(\chi+\frac{\lambda_{2}}{2} \sigma^{2}\right)^{2}}{\left(k^{2}+M_{r}^{2}\right)^{2}}
\end{aligned}
$$

where the subscript 0 on the integrals refers to zero temperature. The first term is an unobservable vacuum energy and the second corresponds to renormalization of $\mu^{2}$. In the final term, which corresponds to $\mu^{2}$ and $\lambda_{1}$ renormalization, we have inserted a renormalization scale in the form of an infrared cutoff on the integral. Physical results are not sensitive to details of the renormalization scheme.

The renormalization of $\lambda_{1}$ to leading order in $1 / N$ includes all $\lambda_{1}$-dependent corrections, and a subset of the $\lambda_{2}$-dependent corrections. There is no $\lambda_{2}$ renormalization in our approximation (with a purely $\sigma_{0}$ background). The corrections to $\lambda_{1}$ lead to a Landau pole at a sufficiently high scale, reflected in a corresponding pathology in the effective potential at a sufficiently high value of $\sigma$. We avoid this problem by taking $\lambda_{1}$ to be somewhat less than the strong coupling limit at the scale $M_{r}$, which in turn we take to lie just above the momentum scales relevant in the effective potential. Thus the Landau pole is safely out of range at the scales of interest. 
Carrying out the summation in Eq. (6) gives

$$
\begin{aligned}
N^{-2} V(\sigma, \chi) & =\frac{1}{2} \chi \sigma^{2}+\frac{2 \mu^{2} \chi}{\lambda_{1}}-\frac{\chi^{2}}{\lambda_{1}}+\frac{\chi^{2}}{64 \pi^{2}}\left(\ln \frac{\chi}{M_{r}^{2}}-\frac{3}{2}\right) \\
& +\frac{T^{4}}{2 \pi^{2}} \int_{0}^{\infty} x^{2} d x \ln \left(1-\exp \left[-\sqrt{x^{2}+\chi / T^{2}}\right]\right) \\
& +\frac{\left(\chi+\frac{\lambda_{2} \sigma^{2}}{2}\right)^{2}}{64 \pi^{2}}\left(\ln \frac{\left(\chi+\frac{\lambda_{2} \sigma^{2}}{2}\right)}{M_{r}^{2}}-\frac{3}{2}\right) \\
& +\frac{T^{4}}{2 \pi^{2}} \int_{0}^{\infty} x^{2} d x \ln \left(1-\exp \left[-\sqrt{x^{2}+\frac{\left(\chi+\frac{\lambda_{2} \sigma^{2}}{2}\right)}{T^{2}}}\right]\right) .
\end{aligned}
$$

The auxiliary field is eliminated by solving its equation of motion

$$
\left(\frac{\partial V(\sigma, \chi)}{\partial \chi}\right)_{\sigma}=0
$$

This transcendental equation, which we solve numerically, is equivalent to a single SchwingerDyson equation for the scalar masses. Had we introduced another auxiliary field to resum the $\lambda_{2}$ interaction, we would have coupled Schwinger-Dyson equations for the scalar masses. Substituting the solution $\chi(\sigma)$ into $V(\sigma, \chi)$ gives the finite temperature effective potential as a function of $\sigma$ alone: $V(\chi(\sigma), \sigma) \equiv U(\sigma)$. We have carried out numerical computations of $U(\sigma)$ for a range of coupling strengths and temperatures.

In Fig. 3 we present numerical results for the choice of couplings $\lambda_{1}\left(M_{r}\right) / 16 \pi^{2}=\lambda_{2} / 16 \pi^{2} \approx 0.25$, where we have taken $M_{r} \approx 4.5 v / N$. This value for the loop expansion parameters is large, but still in a range that keeps the scalar masses, Eq. (舟), somewhat below $4 \pi v / N$, and the Landau pole above the momentum range relevant to the problem. For various temperatures, we plot $N^{-2} U(\sigma)+\left(\pi^{2} / 45\right) T^{4}$ for a $\sigma$ range over which $U$ is real. The purely temperature-dependent second term merely shifts the curves onto the same scale, preserving their shapes and ordering.

For $\sigma$ values somewhat smaller than those shown in the curves, below where the NambuGoldstone propagator mass $\chi$ vanishes, $U(\sigma)$ develops a complex part just as in the $O\left(2 N^{2}\right)$ case. However, with $\lambda_{2} \neq 0$ the propagator masses in our approximation do not include all terms of higher order in $\lambda_{2}$ at leading order in $1 / N$. Goldstone's theorem, applied at a minimum of the effective potential, refers not to such propagator masses calculated at lower order, but instead 


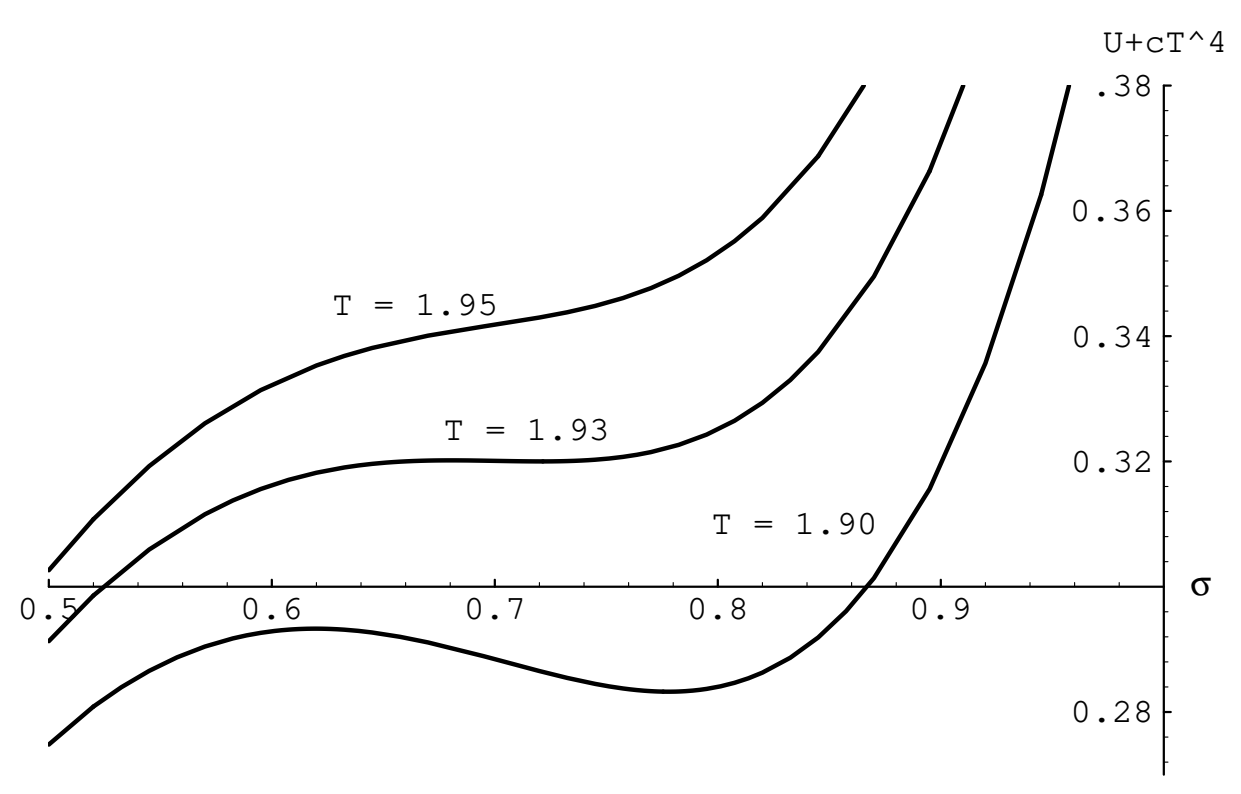

Figure 3: The effective potential $N^{-2} U(\sigma)$ for several temperatures, shifted by $\left(\pi^{2} / 45\right) T^{4}$ for clarity, with all dimensionful quantities measured in units of $v / N$. A first order phase transition must occur below the temperature $T_{\text {inflect }}=1.93 \mathrm{v} / \mathrm{N}$ where the potential maximum and minimum coincide. The field strength discontinuity is at least $0.7 \mathrm{v} / N$ here, with parameters $\lambda_{1}\left(M_{r}=\right.$ $4.54 v / N)=40=\lambda_{2}$.

to second derivatives in symmetry directions. Thus $\chi$ can vanish closer to the origin than the symmetry-breaking minimum. With the parameters of Fig. 3 , $T / \sqrt{\chi}$ is close to unity over the $\sigma$ range shown; we thus avoid infrared divergences at the minimum.

The strongly first order character of the phase transition is apparent from the curves, in which the symmetry-breaking local minimum first appears at a field value $\sigma_{\min } \approx 0.7 v / N$, at temperature $T_{\text {inflect }}$ just below $2 v / N$. With decreasing temperature the minimum evolves to larger field values until it becomes the usual $T=0$ symmetry-breaking vacuum with $\sigma_{\min }=v / N$. We have checked that as $\lambda_{2}$ decreases, $\sigma_{\min }\left(T_{\text {inflect }}\right)$ also decreases, with $T_{\text {inflect }}$ varying only slightly. This behavior is consistent with the second order nature of the phase transition in the $\lambda_{2}=0$ limit.

We can gain some qualitative insight into the origin of first order behavior in the $\lambda_{2} \neq 0$ theory by contrasting it with the $\lambda_{2}=0$ theory. There, all but one of the degrees of freedom are NambuGoldstone modes, whose mass $\chi$ vanishes at the potential minimum $\sigma_{\min }$. The vanishing of $\chi$ leaves Eq. (9) at $\sigma_{\min }$ in the simple form $\sigma_{\min }^{2}-(v / N)^{2}+T^{2} / 6=0$, clearly showing second order 
behavior at $T=T_{c} \equiv \sqrt{6} v / N$. The relatively small masses generated by standard model gauge interactions would in fact turn these modes into pseudo-Nambu-Goldstone bosons, but such small perturbations induce at most weakly first order behavior. Even without those interactions, for small nonzero $\lambda_{2}$ the $\left(N^{2}-1\right) \sigma_{a}$ modes may be thought of as pseudo-Nambu-Goldstone bosons of the $O\left(2 N^{2}\right)$ theory, and their masses do not vanish with the $\pi_{a}$ mass. For large $\lambda_{2}$, however, this is a large perturbation, preventing the equation for $\sigma_{\min }$ from taking on the simple second order form above. The full transcendental equation in our case leads to strongly first order behavior.

\section{Conclusions}

We have argued using an effective linear sigma model that the high temperature electroweak phase transition, in the presence of a strongly interacting symmetry-breaking sector, is strongly first order. With the global symmetry of this sector taken to be $U(N) \times U(N)$, the effective linear theory contains two dimensionless coupling constants $\lambda_{1}$ and $\lambda_{2}$, which are taken to approach the strong coupling limit. The range of couplings considered avoids the Landau pole problem, and corrections to the linear theory, represented by higher-dimensional operators, should be relatively small. Equivalently, a finite ultraviolet cutoff on the theory can be taken to lie above the momentum range of interest. When the strong coupling limit is reached (loop expansion parameters equal unity), higher-dimensional operators are not obviously suppressed and the scalars described by the linear model are not obviously lighter than other new physics. Nevertheless, the strongly first order character of the transition as the couplings of the linear theory approach unit strength provides

evidence that a theory such as technicolor will indeed give rise to a strongly first order electroweak phase transition.

A $1 / N$ expansion provides the framework for the computation. To leading order in $1 / N$, all contributions from the $\lambda_{1}$ interaction can be summed. With respect to $\lambda_{2}$, all planar graphs contribute to leading order in $1 / N$, and only a restricted set of contributions were summed. We argued that this is adequate to determine the strongly first order character of the phase transition. The strength of the transition decreases with $\lambda_{2}$, consistent with a second order transition in the 
$O\left(2 N^{2}\right)$ theory.

The momentum scales in the strongly first order case are such that there is no reason to expect that a high temperature expansion should be reliable, or that the phase transition should be governed by an effective three-dimensional theory. Our results are nevertheless consistent with the hints provided by analysis of such theories [11, 15].

This work may be relevant to the problem of electroweak baryogenesis. More detailed calculations will be necessary to study the dynamics of departure from thermal equilibrium during the transition, but our strongly first order transition provides at least a necessary condition for baryon number generation. Furthermore, the sphaleron mass in our model is the same as the minimal standard model formula [30], $m_{\text {sphaleron }}=(4 \pi K / g)\left\langle\sigma_{0}\right\rangle$, where $K \sim 3$ is a function of the scalar couplings. Since $T_{c}$ is of order $2 v / N$, a strongly first order phase transition to a broken phase with $\left\langle\sigma_{0}\right\rangle / v \sim 1$ ensures Boltzmann suppression of baryon number destruction during and after the phase transition.

\section{Acknowledgments}

We thank Peter Arnold, Sekhar Chivukula, Andy Cohen, Nick Evans, Stephen Hsu, Krishna Rajagopal, Yue Shen, John Terning, and Larry Yaffe for useful discussions and criticism. T.W.A. and S.B.S. thank the Aspen Center for Physics for its hospitality and productive research setting. This work was supported in part under U.S. Department of Energy contract No. DE-AC02-ERU3075. 


\section{References}

[1] A.G. Cohen, D.B. Kaplan and A.E. Nelson, hep-ph/9302210, Annu. Rev. Nucl. Part. Phys. 43, 27 (1993).

[2] D.A. Kirzhnits and A.D. Linde, Ann. Phys. (N.Y.) 101, 195 (1976); M. Dine, R.G. Leigh, P. Huet, A. Linde and D. Linde, hep-ph/9203203, Phys. Rev. D 46, 550 (1992); G.W. Anderson and L.J. Hall, Phys. Rev. D 45, 2685 (1992); G. Amelino-Camelia and S.-Y. Pi, hepph/9211211, Phys. Rev. D 47, 2356 (1993); M. Alford and J. March-Russell, hep-ph/9308364, Nucl. Phys. B417, 527 (1994); N. Tetradis and C. Wetterich, hep-ph/9309257, Int. J. Mod. Phys. A 9, 4029 (1994); P. Arnold and L. Yaffe, hep-ph/9312221, Phys. Rev. D 49, 3003 (1994). Ref. [1] cites and summarizes a voluminous literature.

[3] P. Arnold and O. Espinosa, hep-ph/9212235, Phys. Rev. D 47, 3546 (1993).

[4] S. Weinberg, Phys. Rev. D 9, 3357 (1974).

[5] Z. Fodor, J. Hein, K. Jansen, A. Jaster and I. Montvay, hep-lat/9409017, Nucl. Phys. B439, 147 (1995); Z. Fodor, J. Hein, K. Jansen, A. Jaster, I. Montvay and F. Csikor, hep-lat/9405021, Phys. Lett. B 334, 405 (1994).

[6] R.V. Gavai, U.M. Heller, F. Karsch, T. Neuhaus and B. Plache, hep-lat/9207033, Phys. Lett. B 294, 84 (1992); K. Jansen and P. Seuferling, Nucl. Phys. B343, 507 (1990); C. Frick, K. Jansen, J. Jersak, I. Montvay, P. Seuferling and G. Munster, Nucl. Phys. B331, 515 (1990); N. Tetradis and C. Wetterich, Nucl. Phys. B398, 659 (1993).

[7] V. Jain, hep-ph/9205232, Nucl. Phys. B394, 707 (1993).

[8] P. Ginsparg, Nucl. Phys. B170, 388 (1980).

[9] F. Karsch, hep-lat/9401008, in Lattice '93, Proceedings of the International Symposium, Dallas, Texas, ed. T. Draper et al. [Nucl. Phys. B (Proc. Suppl.) 34, 63 (1994)]. See also references in S. Gottlieb, ibid. 20, 247 (1991). 
[10] Some finite temperature aspects of the nonlinear theory have been considered in the literature, requiring however a replacement for the lack of an explicit order parameter; see e.g. P. Gerber and H. Leutwyler, Nucl. Phys. B321, 387 (1989); H. Meyer-Ortmanns, H.J. Pirner and A. Patkos, Phys. Lett. B 295, 255 (1992).

[11] R.D. Pisarski and F. Wilczek, Phys. Rev. D 29, 338 (1984).

[12] Z. Frei and A. Patkos, Phys. Lett. B 247, 381 (1990); D. Metzger, H. Meyer-Ortmanns and H.J. Pirner, hep-ph/9312252, Phys. Lett. B 321, 66 (1994) (see other references therein); erratum, Phys. Lett. B 328, 547(E) (1994).

[13] S. Raby, Phys. Rev. D 13, 2594 (1976), and references therein.

[14] This is the scale above which a nonlinear, low energy effective theory (not including the scalars) must break down. See M. Soldate and R. Sundrum, Nucl. Phys. B340, 1 (1990).

[15] A.J. Paterson, Nucl. Phys. B190, 188 (1981).

[16] Y. Shen, hep-lat/9303014, Phys. Lett. B 315, 146 (1993); Y. Shen, hep-lat/9311018.

[17] S. Coleman and E. Weinberg, Phys. Rev. D 7, 1888 (1973).

[18] H. Yamagishi, Phys. Rev. D 23, 1880 (1980); D.J. Amit, Field Theory, the Renormalization Group, and Critical Phenomena, revised 2nd ed. (World Scientific, Singapore, 1989) p. 337.

[19] L. Dolan and R. Jackiw, Phys. Rev. D 9, 3320 (1974).

[20] R. Jackiw, Phys. Rev. D 9, 1686 (1974).

[21] J. Hubbard, Phys. Rev. Lett. 3, 77 (1959); R.L. Stratanovich, Sov. Phys. Dokl. 2, 416 (1958); S. Coleman, R. Jackiw and D. Politzer, Phys. Rev. D 10, 2491 (1974); R. Root, Phys. Rev. D 10, $3322(1974)$.

[22] M. Carena and C.E.M. Wagner, hep-ph/9209233, Nucl. Phys. B407, 606 (1993).

[23] M. Reuter, N. Tetradis and C. Wetterich, hep-ph/9301271, Nucl. Phys. B401, 567 (1993). 
[24] P. Fendley, Phys. Lett. B 196, 175 (1987).

[25] C.G. Boyd, D.E. Brahm and S.D.H. Hsu, hep-ph/9206235, Phys. Rev. D 48, 4952 (1993); hep-ph/9304254, Phys. Rev. D 48, 4963 (1993).

[26] J.R. Espinosa, M. Quiros and F. Zwirner, Phys. Lett. B 291, 115 (1992).

[27] E. Weinberg and A. Wu, Phys. Rev. D 36, 2474 (1987).

[28] K. Symanzik, Commun. Math. Phys. 16, 48 (1970); J. Iliopoulos, C. Itzykson and A. Martin, Rev. Mod. Phys. 47, 165 (1975); Y. Fujimoto, L. O'Raifeartaigh and G. Parravicini, Nucl. Phys. B212 268 (1983); A. Dannenberg, Phys. Lett. B 202, 110 (1988).

[29] A.H. Guth and S.-Y. Pi, Phys. Rev. D 32, 1899 (1985); K.A. Olive, Phys. Rep. 190, 307 (1990).

[30] F.R. Klinkhamer and N.S. Manton, Phys. Rev. D 30, 2212 (1984). 